\title{
Extracting Figures and Captions from Scientific Publications
}

\author{
Pengyuan Li, Xiangying Jiang, Hagit Shatkay \\ Department of Computer \& Information Sciences, University of Delaware \\ Newark, Delaware, USA \\ \{pengyuan,jiangxy,shatkay\}@udel.edu
}

\begin{abstract}
Figures and captions convey essential information in scientific publications. As such, there is a growing interest in mining published figures and in utilizing their respective captions as a source of knowledge. There is also much interest in image captioning systems that can automatically generate captions for images, whose training requires large datasets of image-caption pairs. Notably, the first fundamental step of obtaining figures and captions from publications is neither well-studied nor yet well-addressed. In this paper, we introduce a new and effective system for figure and caption extraction, PDFigCapX. Unlike current methods that extract figures by handling raw encoded contents of PDF documents, we separate text from graphical contents and utilize layout information to detect and disambiguate figures and captions. Files containing the figures and their associated captions are then produced as output to the enduser. We test PDFigCapX on both a previously used generic dataset and on two new sets of publications within the biomedical domain Our experiments and results show a significant improvement in performance compared to the state-of-the-art, and demonstrate the effectiveness of our approach. Our system will be available for use at: https://www.eecis.udel.edu/ compbio/PDFigCapX.
\end{abstract}

\section{CCS CONCEPTS}

- Information systems $\rightarrow$ Data extraction and integration; • Applied computing $\rightarrow$ Document analysis;

\section{KEYWORDS}

Data extraction; scientific document analysis; figure extraction; caption extraction; PDF parsing

\section{ACM Reference Format:}

Pengyuan Li, Xiangying Jiang, Hagit Shatkay. 2018. Extracting Figures and Captions from Scientific Publications. In 2018 ACM Conference on Information and Knowledge Management (CIKM'18), October 22-26, 2018, Torino, Italy. ACM, New York, NY, USA, 4 pages. https://doi.org/10.1145/3269206.3269265

\section{INTRODUCTION}

Figures and captions convey essential information in scientific publications. As such, there is a growing interest in mining figures and captions appearing within scientific publications [1,2]. Several platforms, such as the Yale Image Finder [3] and SourceData [4], aim to enable users to search for relevant figures and captions. There is also much current interest in automatically generating

Permission to make digital or hard copies of part or all of this work for personal or classroom use is granted without fee provided that copies are not made or distributed for profit or commercial advantage and that copies bear this notice and the full citation on the first page. Copyrights for third-party components of this work must be honored

For all other uses, contact the owner/author(s).

CIKM '18, October 22-26, 2018, Torino, Italy

(C) 2018 Copyright held by the owner/author(s).

ACM ISBN 978-1-4503-6014-2/18/10.

https://doi.org/10.1145/3269206.3269265 image descriptions, namely, image-captioning. Training captioning systems can benefit from having large collections of figure-caption pairs. However, the first step toward these goals, namely, extracting figure and caption pairs from scientific publications, is neither wellstudied nor yet well-addressed. We thus introduce an effective new method to extract figures and captions from scientific publications.

Notably, most current scientific publications are stored in Portable Document Format (PDF), in which figures are encoded as raster graphics (e.g. PNG, JPEG) or vector graphics (e.g. SVG, EPS). Quite a few tools (e.g. PDFBox [6], Xpdf [7]) convert PDF documents into a structured XML/HTML format while extracting figures. As these tools do not distinguish figure captions from the rest of the text in the article, they do not associate figures with their respective captions. Moreover, they often extract individual components in the figure, rather than the complete figure as a whole. For instance, a vector graphics figure, which consists of numerous line fragments and dots, will be broken into many images corresponding to the individual parts. Thus, these tools leave much room for improvement in extracting figures or figure-caption pairs.

Specific approaches aiming to extract figures and captions from PDF documents have been recently proposed. Lopez et al. [8] and Choudhury et al. [9] introduced methods based on available tools (Xpdf and PDFBox respectively), but neither method handles vector graphics within scientific publications.

Identifying vector graphics that form actual figures is challenging because vector graphics may represent graphical objects that are not figures, such as the border line at a page's margin or mathematical symbols within the document. Limited methods handling such figures have been proposed before [10] for extracting figures (e.g. line graphs, bar charts etc.) consisting of simple geometric components, but have not been generalized to more complex figures. More recent methods, typically based on multiple domainspecific heuristics, have been developed for specific areas, such as high-energy physics (PDFPlotExtractor [11]) and computer science (pdffigures2 [12]). While these tools aim to separate vector graphics that constitute figures from those that do not, via clustering and classification, vector graphics are often incorrectly extracted due to the complex figure and document structure.

Here we present a new and effective scheme for extracting figures and associated captions from scientific publications, which also proves to work well across a variety of scientific domains. Unlike earlier methods, our method does not directly parse the raw graphical objects encoded in the PDF (raster graphics and vector graphics). Rather, it first separates the text contents from the graphical contents of the PDF file, and applies Connected Component Analysis (CCA $[2,5]$ ) to the graphical contents in order to detect individual figures. Figure-caption pairs are then recovered by processing layout-information of the PDF as well as the text-part extracted from the PDF file. 
The rest of the paper is organized as follows: Sec. 2 introduces the method itself; in Sec. 3 we present our experiments and discuss the results; Sec. 4 concludes and outlines future work.

\section{METHODS}

Our goal is to extract figures and captions from scientific publications. The complete framework for our approach is summarized in Fig. 1. The rest of this section provides detail about each of the steps.

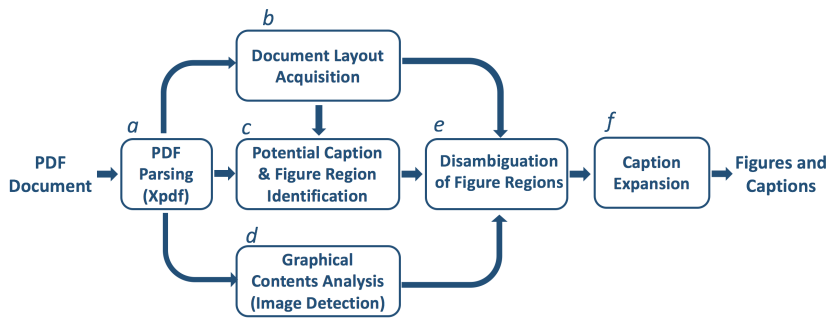

Figure 1: Our framework for figure and caption extraction.

a. PDF parsing: We parse the input PDF document using the publicly available tool, $X p d f$ [7], which partitions the file contents into textual contents stored in an HTML file as text objects, and graphical contents stored as text-stripped image-pages containing only the graphics from the original file. Each text object in the HTML corresponds to a text line in the PDF document, along with position information (staring point coordinates, font type and size, etc.) indicating the line's exact starting position and size within the PDF document. The position and length of each text line in the original PDF can thus be estimated using the stored information. Fig. 2 shows the result of the initial parsing process applied to a PDF page. b. Document layout acquisition: As published documents typically adhere to certain appearance and organization conventions, we identify several layout parameters to guide the extraction process:

Text Line Height and Width: As text within the body of the paper typically has a characteristic font size, the text line height, $l_{\text {height }}$, and width, $l_{\text {width }}$, are set to be the most common (mode) height and width among all text objects stored in the HTML file.

Contents Region: This is the total region of the page in which any textual or figure contents can appear, and is typically located within well-defined margins. The contents region is represented as the coordinates $\left[x_{l t}, y_{l t}, x_{r b}, y_{r b}\right]$, where $\left(x_{l t}, y_{l t}\right)$ and $\left(x_{r b}, y_{r b}\right)$ denote the leftmost top point and the rightmost bottom point, respectively, among the positions of all extracted text lines. The width of the contents region, $c_{\text {width }}$, is set to $x_{l t}-x_{r b}$.

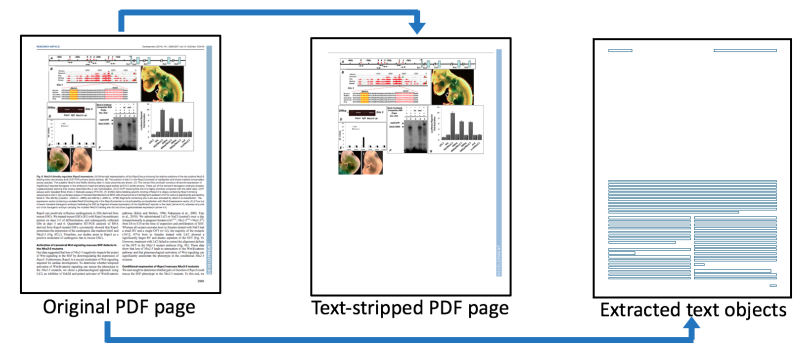

Figure 2: An example of a PDF page parsed using $X p d f$. The original PDF page, shown on the left, is the $7^{\text {th }}$ page in [13].
Number of Columns: The number of columns within a page has much impact on figure location and appearance. For instance, figures in single-column documents often span the whole page and as such are wider than figures appearing in multi-column documents. The number of columns, denoted $\operatorname{col}_{n o}$, is calculated as: $\operatorname{col}_{\text {no }}=$ floor $\left(c_{\text {width }} / l_{\text {width }}\right)$.

c. Identification of potential caption and figure regions: We begin by detecting a putative bounding box for each potential caption, utilizing the text information stored in the structured HTML file. As captions typically start with the prefix Fig or FIG, we consider each text line starting with such a prefix as a potential caption. For each potential caption, $C a p_{i}$, we record its position as it is recorded in the HTML file, and initially set the width of its bounding box to be the length of its first text line.

The figure region typically lies in close proximity to the caption region, and its top/bottom boundaries subsume those of the caption. We thus use the potential caption location to identify potential figure locations. For a single-column page, with a single potential caption in it, $\mathrm{Cap}_{i}$, the potential figure region, region ${ }_{i}$, is set to the whole contents region. If the page contains multiple potential captions, the height of the potential region region $_{i}$, associated with $C a p_{i}$, spans from the position of the previous caption $C a p_{i-1}$ to that of $C a p_{i}$; the width remains that of the contents region. The figure-region associated with the bottom-most caption in the page includes the area all the way to the bottom of the contents region.

For multicolumn documents, the process is similar; the only difference is in the determination of the potential figure width. If the potential caption spans across the center of the page, the figure is assumed to span the whole page width, and as such the width is set to $c_{w i d t h}$; otherwise, the width is set to the width of a single line within the column, $l_{\text {width }}$. Fig. 3 illustrates examples of potential caption and figure regions identified using step $c$.

d. Graphical contents analysis: Fig. 4 illustrates the graphical analysis process. To detect the actual figures, we first detect their constituent parts in each text-stripped PDF page, by employing Connected Component Analysis (CCA). Typically, graphical contents within PDF pages consists of non-white areas. As such, we first generate a binary mask $M$ to separate foreground from background, by applying a threshold $t$ on text-stripped PDF page. Pixels whose intensities are greater than $t$ are set to 1 (white), i.e. background, while all other pixels constitute the foreground are thus set to 0 (black). In our experiments the threshold $t$ is set to 0.95 . Based on the mask $M$ we identify connected components that constitute the actual figure contents by applying the Connected Component Labelling
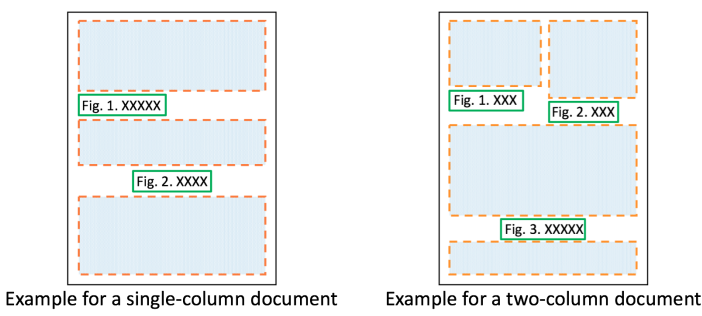

Figure 3: Identification of potential caption and figure regions. Potential figure regions are shown as large dashed boxes; Potential captions are shown as small solid boxes. 

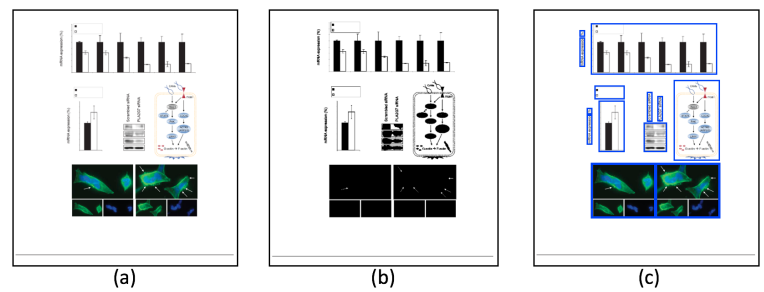

Figure 4: Graphical contents analysis: (a) The text-stripped version of the $6^{\text {th }}$ page in [15]; (b) The binary mask $M$ in which all foreground is shown in black and all background in white; (c) The connected components (each shown within a blue bounding box) detected by our method.

method [14]. We then set a bounding box around each connected component, encompassing the smallest rectangle containing all the pixels within the component.

e. Disambiguation of figure regions: As demonstrated by Fig. 4, the previous step results in relatively small figure-constituents, which may combine to form one or more individual complete figures. To unambiguously identify the actual individual figures within each potential figure region region $_{i}$, we merge all bounding boxes of connected components within each potential region into a single figure denoted $f i g_{i}$. We note that text-elements that are essential parts of some figures (e.g. figure legends, or axes labels in graphs), are initially removed and stored in an HTML file as text objects during the parsing step (Step $a$ ). To recover the textual contents and place it back into the figure, we merge each figure $f i g_{i}$ with all the text objects located close to it. Fig. 5 provides an example of the figure disambiguation process.

f. Caption expansion: As discussed in Step c, headers of potential captions that can help locate associated figures are first detected using specific prefixes. To expand the header into a complete caption, we detect the continuous text block following the potential header. The text block is defined as a set of consecutive text objects (recorded in the HTML file), where the last object ends with a period and its width is lower than that of the bounding box surrounding $C a p_{i}$, or where the last object is followed by a vertical gap.

The region of a caption is estimated by merging the bounding boxes of all text objects within the text block. The complete caption associated with $f i g_{i}$ is formed by combining the text content of all text objects from top to bottom within the detected text block. The

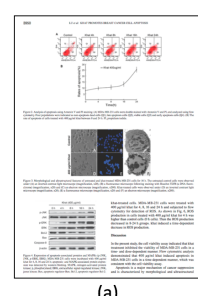

(a)

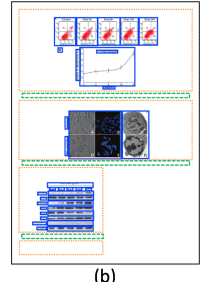

(b)

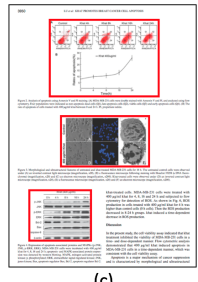

Figure 5: An example of the figure disambiguation process. (a) The original PDF page $\left(4^{\text {th }}\right.$ page in [16]). (b) Connected components are shown in blue solid boxes; potential captions in green dashed boxes; potential figure regions in dotted orange boxes. (c) Figure regions (shown in red solid boxes) detected by our disambiguation process. complete figure and its associated caption are then stored as part of the output files, along with their position on the source PDF page.

\section{EXPERIMENTS}

\subsection{Experimental settings}

To evaluate our method, we compare the performance of our system to that of two state-of-the-art systems, pdffigures2 [12] and PDFPlo$t$ Extractor [11], performing three tasks: figure extraction, caption extraction, and the combined task of figure-caption pair extraction.

Three datasets are used in this study. The first, denoted CS-150, was introduced in [12]. It comprises 150 publications selected from top computer science conferences.To test our tool on more general and more challenging scientific papers, we use publications from the biomedical literature where complex figures are often used to show experimental results. The first dataset we created, denoted GXD-200, comprises 200 documents, retaining the same year range as in the CS-150 dataset. Noting that PDF coding varies significantly between older and newer PDF versions, which can impact the extraction performance, the second dataset we created, denoted PMC-200, spans a larger range of years, namely 1990-2017. Table 1 provides statistics for all three datasets.

To evaluate our extraction performance, we use the standard evaluation metrics of precision and recall, defined as:

$$
\begin{gathered}
\text { Precision }=\frac{\# \text { of figures (and/or captions) correctly extracted }}{\text { \# of figures (and/or captions) extracted }}, \\
\text { Recall }=\frac{\# \text { of figures (and/or captions) correctly extracted }}{\# \text { of figures (and/or captions) in the ground-truth }},
\end{gathered}
$$

A figure or a caption is considered to be correctly extracted if the overlap-ratio between the respective bounding boxes, defined as:

$$
\frac{\text { Area[(bound. box detected }) \cap(\text { ground-truth bound. box })]}{\text { Area[(bound. box detected) } \cup \text { (ground-truth bound. box })]} \text {, }
$$

is greater than $3 / 5$ [19]. A figure-caption pair is deemed to be correctly extracted when the figure and the caption individually are both correctly extracted.

\subsection{Results and discussion}

Fig. 6(A) summarizes the results for the figure extraction task. The pdffigures2 system - which was specifically built for the computer science domain -indeed attains the highest precision and recall on the CS-150 dataset. Our method, which is not specifically designed for this domain, still attains $93.50 \%$ precision and $88.00 \%$ recall, outperforming the PDFPlotExtractor system. When applied to the GXD-200 dataset, our method attains a significantly higher recall $(93.03 \%)$ than that of the two other systems $(65.91 \%, 69.89 \%)$ for figure extraction. We note that while the precision attained by

Table 1: Statistics for each of the datasets used in our experiments. The \# of figures may exceed that of captions as a figure may appear on a page without an associated caption.

\begin{tabular}{ccccccc}
\hline $\begin{array}{c}\text { Dataset } \\
\text { Name }\end{array}$ & $\begin{array}{c}\text { Year } \\
\text { Range }\end{array}$ & $\begin{array}{c}\text { Data } \\
\text { Source }\end{array}$ & $\begin{array}{c}\text { \# of } \\
\text { Docs }\end{array}$ & $\begin{array}{c}\text { \# of } \\
\text { Figures }\end{array}$ & $\begin{array}{c}\text { \# of } \\
\text { Captions }\end{array}$ & $\begin{array}{c}\text { \# of Fig } \\
\text {-Cap Pairs }\end{array}$ \\
\hline CS-150 & $2009-2014$ & {$[12]$} & 150 & 458 & 458 & 458 \\
GXD-200 & $2009-2014$ & GXD[17] & 200 & 1335 & 1298 & 1298 \\
PMC-200 & $1990-2017$ & PMC[18] & 200 & 1042 & 1032 & 1032 \\
\hline
\end{tabular}




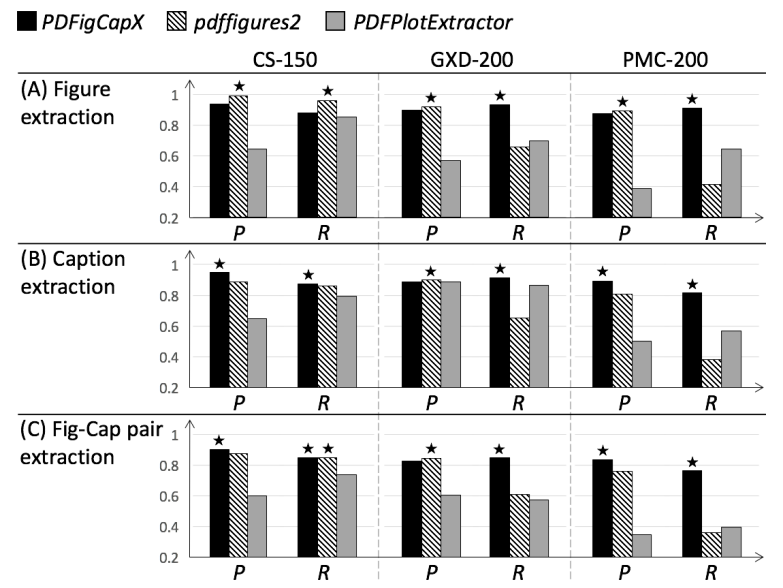

Figure 6: Precision $(P)$ and recall $(R)$ obtained by our system (left solid black column), compared with those obtained by the other two state-of-the-art systems. The highest values attained are indicated by a star.

our method over this dataset is slightly lower (by $2 \%$ ) than that of pdffigures2, our recall is much higher (by $27 \%$ ).

All three systems show the lowest performance on the more general, PMC-200 dataset; this decline is much more pronounced in pdffigures2 and PDFPlotExtractor. Notably, the PMC-200 dataset contains articles published during the period 1990-2017. As both pdffigures2 and PDFPlotExtractor rely on handling the encoded raw graphical objects, the performance of these systems is adversely impacted by the PDF coding variations. In contrast, our system attains a significantly higher recall (90.79\%) than that of the other two systems $(41.47 \%, 64.49 \%)$ on the same dataset, with only a slight loss $(1.65 \%)$ in precision compared to pdffigures2. These results demonstrate that our method provides an effective and robust means for figure extraction from PDF files.

As seen in Fig. 6(B), our method attains the highest precision and the highest recall for caption extraction over both the CS-150 and the PMC-200 datasets. Notably, over the GXD-200 dataset, the precision of our method (88.74\%) is slightly lower than that of pdffigures $2(90.01 \%)$, but our recall is again significantly higher (91.14\% compared with $65.24 \%$ ).

For caption extraction, the decline in performance of all three systems over the PMC-200 dataset is more pronounced than it is for figure extraction. Among the PMC-200 articles, 34 were older documents published in 1990-2008, and 10 of those are scanned PDF documents. Such scanning, which involves optical character recognition of the text, often leads to errors that make captions more difficult to detect. Thus, for caption extraction, pdffigures2 shows a $38.18 \%$ recall, while PDFPlotExtractor's recall is at $56.98 \%$. In contrast, our method shows a significantly higher recall $(81.40 \%)$ in the face of these issues. Our system thus proves to be much more resilient and reliable in identifying captions in a broad range of PDF files compared to currently available systems.

Last, Fig. 6(C) shows the results for the combined task of figurecaption pair extraction. All three methods show lower performance on this task as it requires both the figure and its respective caption to be correctly extracted. Over the CS-150 dataset, our method still attains the highest precision and the same (highest) recall as that attained by $p d f f i g u r e s 2$. The lower performance is more pronounced in the results obtained on the GXD-200 and PMC-200 datasets as the figure-caption organization in biomedical publications is more complex than that in computer science publications. Over the PMC-200 dataset, our recall and precision are significantly higher than those of the other two methods. Over the GXD-200 dataset, the precision of our method $(82.52 \%)$ is again slightly lower than that of pdffigures2 $(84.45 \%)$, while retaining a significantly higher recall $(84.75 \% \mathrm{vs,}$ $60.6 \%$ ). These results again demonstrate and validate our method as an effective and robust means for extracting figure-caption pairs.

\section{CONCLUSIONS}

We presented a new system, PDFigCapX, for extracting figures and captions from scientific publications. Unlike current methods that extract figures by handling raw encoded contents of PDF documents, our system separates text from graphical contents and utilizes layout information to detect and disambiguate figures and captions. Extensive experiments and results demonstrate that our system is highly effective in terms of both precision and recall. Moreover, PDFigCapX retains its good performance on a broad range of publications varying in topic, style, publication year and overall organization.

Several challenging cases remain as future work, such as identifying the relatively rare cases in which figure-caption pairs span more than a single page.

\section{ACKNOWLEDGMENTS}

This work was partially supported by NIH/NLM awards R56LM011354A and R01LM012527.

\section{REFERENCES}

[1] Ahmed, Z. et al. (2016) Mining biomedical images towards valuable information retrieval in biomedical and life sciences. Database, 2016, baw118.

[2] Shatkay, H., et al. (2006) Integrating image data into biomedical text categorization. Bioinformatics, 22(14), e446-e453.

[3] Xu, S. et al. (2008) Yale Image Finder (YIF): a new search engine for retrieving biomedical images. Bioinformatics, 24(17), 1968-1970.

[4] Liechti, R., et al. (2017) SourceData: a semantic platform for curating and searching figures. Nature methods, 14(11), p.1021.

[5] Li, P. et al. (2018) Compound image segmentation of published biomedical figures. Bioinformatics, 34(7), 1192-2299.

[6] Apache PDFBox 2.0.8. (2017) Available at: www.pdfbox.apache.org.

[7] Noonburg, D. Xpdf 4.0. (2017) Available at: www.xpdfreader.com.

[8] Lopez, L.D. et al. (2011) An automatic system for extracting figures and captions in biomedical pdf documents. In Proc. of IEEE BIBM, 578-581.

[9] Choudhury, S.R. et al. (2013) Figure metadata extraction from digital documents. In Proc. of IEEE ICDAR, 135-139.

[10] Shao, M. and Futrelle, R.P. (2006) Recognition and classification of figures in PDF documents. In Proc. of GREC, 231-242.

[11] Praczyk, P.A. et al. (2013) Automatic extraction of figures from scientific publications in high-energy physics. Information Technology and Libraries, 32(4), 25.

[12] Clark, C. and Divvala, S. (2016) Pdffigures 2.0: Mining figures from research papers. In Proc. of IEEE/ACM JCDL, 143-152.

[13] Cambier, L., et al. (2014) Nkx2-5 regulates cardiac growth through modulation of Wnt signaling by R-spondin3. Development, 141(15), 2959-2971.

[14] Gonzalez, R.C., et al. (2002) Digital Image Processing.

[15] Vainio, P., et al. (2011) Phospholipase PLA2G7, associated with aggressive prostate cancer, promotes prostate cancer cell migration and invasion and is inhibited by statins. Oncotarget, 2(12), p.1176.

[16] Lu, Y., et al. (2017) Khat promotes human breast cancer MDA-MB-231 cell apoptosis via mitochondria and MAPK-associated pathways. Oncology letters, 14(4), 3947-3952.

[17] Finger, J.H., et al. (2017) The mouse gene expression database (GXD): 2017 update. Nucleic Acids Research, 45(D1), D730-D736.

[18] PMC Open Access Subset. (2018) Available at: www.ncbi.nlm.nih.gov/pmc

[19] De Herrera, A.G.S, et al. (2013) Overview of the ImageCLEF 2013 Medical Tasks. In CLEF Working Notes: http://ceur-ws.org/Vol-1179. 